\title{
The Simulation of Better Pan
}

\author{
Zhang Bincan \\ Computer Science and Technology Wuhan University of Technology, Wuhan, China \\ zhangbc2012@gmail.com
}

\begin{abstract}
We determined the best pan by two models. At first, we established a prism model with great heat convection on profiles through reasonable simplification. According to thermophysics, we derived partial differential equations to describe temperature field distribution of pans. However, the equations are too complex. We offered decoupled boundary conditions for simplification. But the solutions are beyond our capability. We conducted numerical simulation based on the finite element method. Through ANSYS, we obtained heat distribution of various shapes concluded a trend.

Index Terms - Finite element method; Heat distribution; Temperature filed;
\end{abstract}

\section{Introduction}

In the given problem description, the heat distribution of round and rectangular pans is hinted: when baking in a rectangular pan, heat is concentrated in the 4 corners and the product gets overcooked at the corners while in a round pan the heat is distributed evenly over the entire outer edge and the product is not overcooked at the edges. Following this reasoning, we expect that the heat distribution will become increasingly even as the number of pan edges increases.

In our proving model, we started by the physical phenomenon and gave the heat conduction equations of threedimensional cases. Although we understand how to describe this phenomenon through mathematical language, the analytic solutions of partial differential equations, however, are too complicated for us [1]. Under such circumstance, we turned to the finite element method for numerical solutions. Taking advantage of ANSYS, we successfully simulated the threedimensional temperature filed and presented the image and analysis of our results, testifying our expectation to some extent.

\section{Model 1}

\section{A. TablesModel Establishment}

Equations Derivation of Three-dimensional Temperature Field:

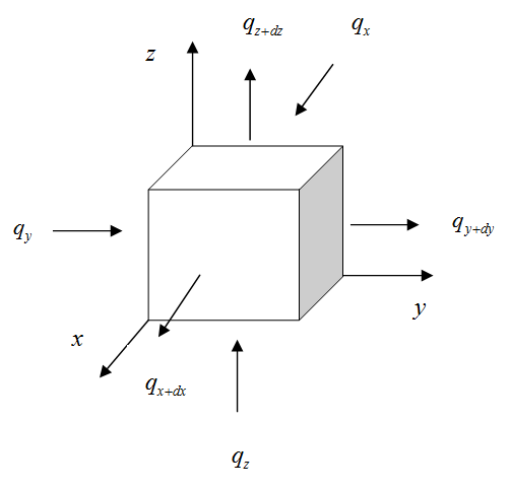

Fig.1.infinitesimal hexahedron
In a simplified prism, we randomly select a infinitesimal hexahedron (Fig.1.). We apply the Fourier Law [6]:

$$
q=-k \frac{\partial T}{\partial n}=-k\left(\frac{\partial T}{\partial x}+\frac{\partial T}{\partial y}+\frac{\partial T}{\partial z}\right)
$$

In the rectangular coordinate system:

$$
q_{z}=-k \frac{\partial T}{\partial z} q_{y}=-k \frac{\partial T}{\partial y} \quad q_{z}=-k \frac{\partial T}{\partial z}
$$

The X-component increased heat in unit time of the infinitesimal area:

$$
\left(q_{x}-q_{x+d x}\right) d y d z=q_{x} d y d z-\left(q_{x}+\frac{\partial q_{x}}{\partial x} d x\right) d y d z=-\frac{\partial q_{x}}{\partial x} d x d y d z=k \frac{\partial^{2} T}{\partial x^{2}} d x d y d z
$$

The Y-component increased heat in unit time of the infinitesimal area:

$\left(q_{y}-q_{y+d y}\right) d x d z=q_{y} d x d z-\left(q_{y}+\frac{\partial q_{y}}{\partial y} d y\right) d x d z=-\frac{\partial q_{y}}{\partial y} d y d x d z=k \frac{\partial^{2} T}{\partial y^{2}} d x d y d z$

The Z-component increased heat in unit time of the infinitesimal area:

$\left(q_{z}-q_{z+d z}\right) d x d y=q_{z} d x d y-\left(q_{z}+\frac{\partial q_{z}}{\partial z} d z\right) d x d y=-\frac{\partial q_{z}}{\partial z} d z d x d y=k \frac{\partial^{2} T}{\partial z^{2}} d x d y d z$

The overall increased heat in unit time of the infinitesimal area:

$k \frac{\partial^{2} T}{\partial x^{2}} d x d y d z+k \frac{\partial^{2} T}{\partial y^{2}} d x d y d z+k \frac{\partial^{2} T}{\partial z^{2}} d x d y d z=k\left(\frac{\partial^{2} T}{\partial x^{2}}+\frac{\partial^{2} T}{\partial y^{2}}+\frac{\partial^{2} T}{\partial z^{2}}\right) d x d y d z$

The necessary heat in unit time for the temperature rise of the infinitesimal area:

$$
c \rho d x d y d z \frac{\partial T}{\partial t}
$$

On the basis of the law of conservation of energy, equating the heat increased with the heat necessary yields:

$$
\begin{gathered}
k\left(\frac{\partial^{2} T}{\partial x^{2}}+\frac{\partial^{2} T}{\partial y^{2}}+\frac{\partial^{2} T}{\partial z^{2}}\right) d x d y d z=c \rho \frac{\partial T}{\partial t} d x d y d z \\
\frac{\partial T}{\partial t}=\frac{k}{c \rho}\left(\frac{\partial^{2} T}{\partial x^{2}}+\frac{\partial^{2} T}{\partial y^{2}}+\frac{\partial^{2} T}{\partial z^{2}}\right)=\alpha^{2}\left(\frac{\partial^{2} T}{\partial x^{2}}+\frac{\partial^{2} T}{\partial y^{2}}+\frac{\partial^{2} T}{\partial z^{2}}\right)
\end{gathered}
$$

The front of the equation is a linear second order partial differential equation, whose solution needs initial and boundary conditions. In our case : The initial condition:

$$
T(x, y, 0)=T_{0}
$$


On the basis of our assumptions, we give the boundary conditions:

Because of the adiabatic property of the upper and lower surfaces, we give the Neumann boundary condition:

$$
\left.\frac{\partial T}{\partial z}\right|_{z=0}=0,\left.\frac{\partial T}{\partial z}\right|_{z=t}=0
$$

According to the heat convection boundary conditions on the four side surfaces, we give the third boundary condition:

$$
-k \frac{\partial T}{\partial n}=h\left(T_{\infty}-T_{\text {sides }}\right)
$$

\section{B. Case Analysis}

We take advantage of ANSYS to conduct numerical simulation to temperature filed of pans[2].

Node selection: 8-node 70 unit.

Meshing: tetrahedral meshing

Parameter selection: after consulting data of ovens and stainless steel, we select the parameter, listed in Table 1.

Table 1 Parameter selection

\begin{tabular}{llll}
\hline $\begin{array}{l}\text { parame } \\
\text { ters }\end{array}$ & meanings & $\begin{array}{l}\text { valu } \\
\text { es }\end{array}$ & units \\
\hline$T_{0}$ & $\begin{array}{l}\text { initial } \\
\text { temperature }\end{array}$ & 80 & {$\left[{ }^{\circ} \mathrm{c}\right]$} \\
$T_{\infty}$ & $\begin{array}{l}\text { air temperature } \\
\text { in the oven }\end{array}$ & 190 & {$\left[{ }^{\circ} \mathrm{c}\right]$} \\
$h$ & $\begin{array}{l}\text { Convective heat } \\
\text { transfer } \\
\text { coefficient }\end{array}$ & 100 & {$\left[\mathrm{Wm} \cdot{ }^{\circ} \mathrm{C}\right]$} \\
& $\begin{array}{l}\text { specific heat } \\
\text { capacity } \\
\text { density }\end{array}$ & 450 & {$\left[\mathrm{~J} / \mathrm{kg} \cdot{ }^{\circ} \mathrm{c}\right]$} \\
$\rho$ & $\begin{array}{l}\text { Thermal } \\
\text { conductivity } \\
k\end{array}$ & 7800 & {$\left[\mathrm{~kg} / \mathrm{m}^{3}\right]$} \\
& Surficial area & 0.038 & {$\left[\mathrm{~W} / \mathrm{m}^{\circ}{ }^{\circ} \mathrm{c}\right]$} \\
\hline \hline
\end{tabular}

Through ANSYS, temperature filed distribution, fitted curves of marginal heat distribution and the temperature at a corner as a function of time ( attached in the appendix) are plotted.

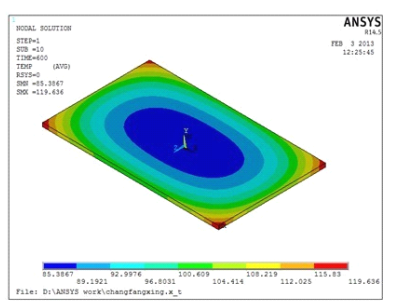

Fig. 2 temperature filed of rectangular pans

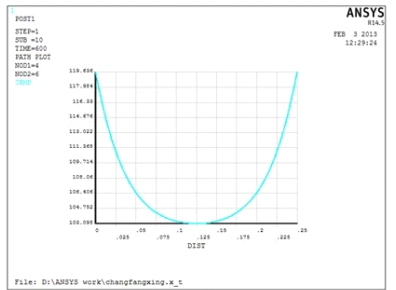

Fig. 3 marginal temperature fitted curve of rectangular pans

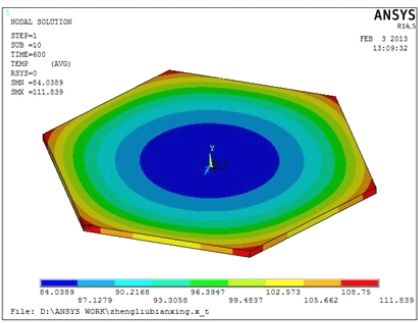

Fig. 4 temperature filed of hexagonal pans

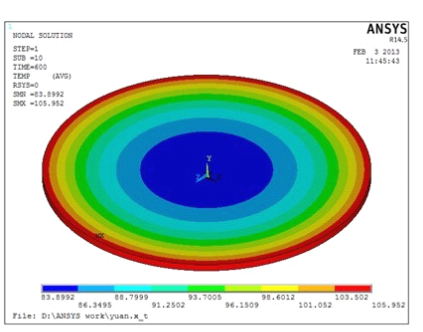

Fig. 6 temperature filed of round pans

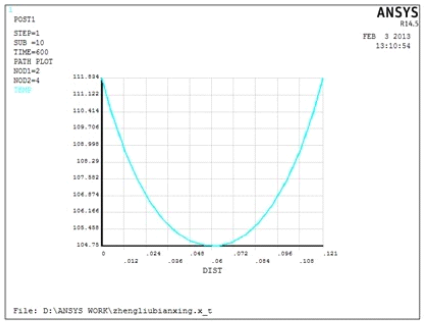

Fig.5 marginal temperature fitted curve of hexagonal pans

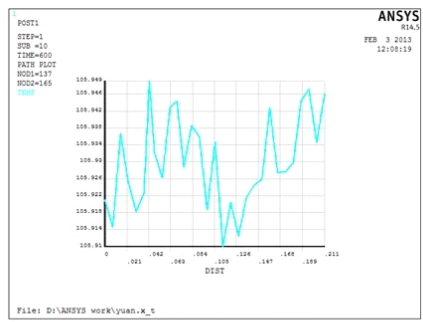

Fig.7 marginal temperature fitted curve of round
C. Results

By Figures above, we draw the following conclusions:

- For any polygon other than round, marginal unevenness of heat distribution does exist and is centered at the corners while the heat distribution of round pans is uniform.

- Fitted curves of marginal heat distribution are always concave, approaching their minimum at the middle of each side and maximum at the end, which means heat at the edge is less than heat at the corner.

- Heat distribution unevenness decreases gradually as $\mathrm{n}$ increases and for a round, heat unevenness is negligible.

- The results of our model meets the phenomenon described in the problem: the heat is distributed evenly over the entire outer edge in a round pan; and in a rectangular pan heat is concentrated in the 4 corners and the product gets overcooked at the corners (and to a lesser extent at the edges). Therefore, the reasonableness of our model is certified to some $(n \geq 4)$ extent.

\section{Model 2}

\section{A. Model Establishment}

According to the second and the third assumptions, we are able to select a pan of the most width to determine the minimum W (width of the oven)[3]. Connecting any two adjacent vertexes and its geometric center of a regular polygon one gets a triangle as Fig. 7 . 


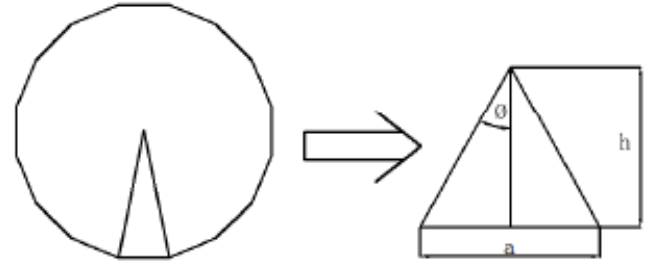

Fig. 8 basic triangle

Assume $h$ is the vertical distance from the center to a side. $a$ is the length of a side. On the basis of geometric features of regular polygon, we have:

$$
\phi=\frac{\pi}{n} \quad a=2 h \tan \phi
$$

Given the pan must have an area of A, we can write:

$$
A=\frac{1}{2} n h a=n h^{2} \tan \frac{\pi}{n} \Rightarrow h=\sqrt{\frac{A}{n \tan \frac{\pi}{n}}}
$$

\section{B. The Min-Width}

When $n(n \geq 4)$ is even, we have the diagonal distance (minimum width $D_{\text {in }}$ )and perpendicular distance between opposite sides(minimum width ), $D_{\text {out }}$ as Fig. 8 .

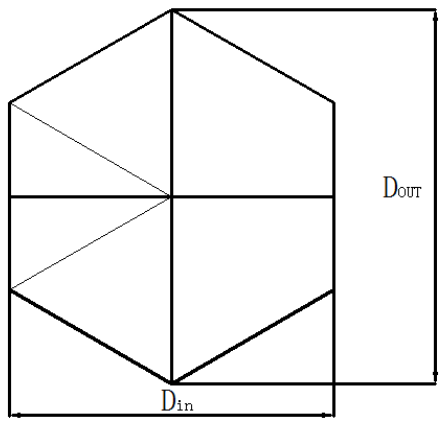

Fig. 9 hexagon

$$
D_{\text {in }}(\text { even })=2 h=2 \sqrt{\frac{A}{n \tan \frac{\pi}{n}}}(n=4,6,8 \ldots)
$$

When $n$ is odd, we can also get $D_{i n}($ odd $)$ the, as shown in Fig. 9.
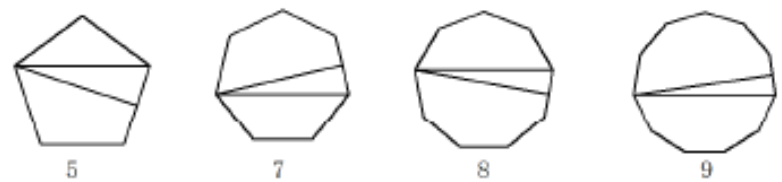

Fig. 10

$$
D_{\text {in }}(o d d)=h+\frac{h}{\cos \frac{\pi}{n}}(\mathrm{n}=5,7,9 \ldots)
$$

According to our assumptions, to put in more pans, we have

$$
N=\left[\frac{L}{D_{\text {in }}}\right]
$$

Fig. 10 shows a comparison between values of $D_{\text {in }}(e v e n)$ and $\cdot D_{\text {in }}(o d d)$

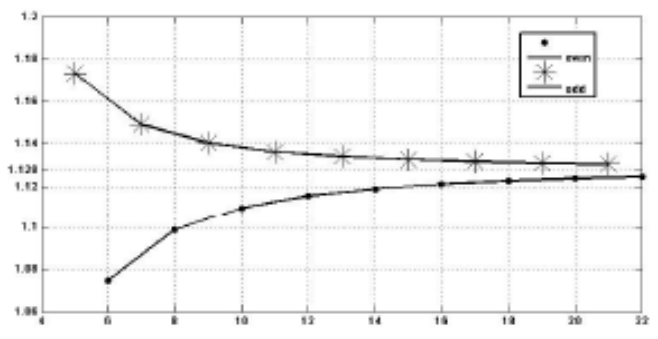

Fig. 11 comparison of odd and even

From the picture, we can safely draw the conclusion: even-edge pans are better in terms of the maximum number. In our subsequent discussion, we study even-edge polygons only.

\section{The Max-Width}

Because pans of any shape should be able to fit into the oven, the width of oven $W$ is not less than the max-width of pans. We give $D_{\text {out }}$ as a function of $n$

$$
D_{\text {out }}=\frac{2 h}{\cos \frac{\pi}{n}}=2 \sqrt{\frac{2 A}{n \sin \frac{2 \pi}{n}}}(k=4,6,8 \ldots)
$$

To determine the greatest width, we plot to show the trend of $D_{\text {our }}$ (maximum width)

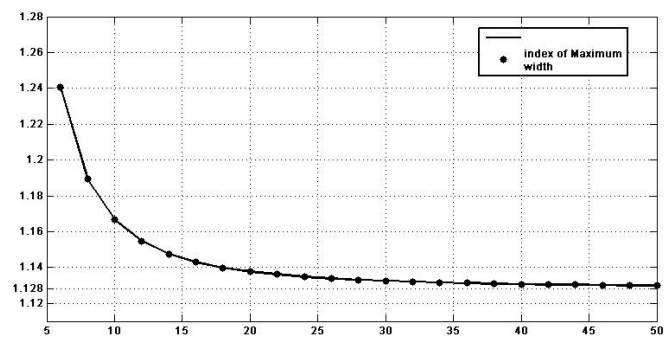

Fig. 12 Index of Maximum width

We see $g(n)$ is a monotone decreasing function (so is as $\frac{L}{D_{\text {in }}}$ ), approaching to $\frac{\sqrt{\pi}}{2} \approx 0.886$ and $g(n) \leq 1$, Again $N$ may be equal to $\left[n_{0}\right]$ but we still have $\left[n_{0}\right] \geq N$.

This means the value of $\mathrm{N}$ gradually decreases as $\mathrm{n}$ increases and the shape of maximum number is square. 
However, we have never discussed the number of rectangular pans.

Assume a width to length ratio of $w / l$ for the rectangular pans. For a maximum number, the length of the pan equals the width of the oven:

$$
l=W, l \times w=A
$$

Then we get the maximum number of rectangular pans:

$$
n_{1}=\frac{L}{w}=\frac{L l}{A}=\frac{L W}{A}=2 L \sqrt{\frac{1}{3 \sin \frac{\pi}{3} A}}
$$

Have $n_{1}$ divided by $n_{0}$ to compare them:

$$
\frac{n_{1}}{n_{0}}=\frac{2 L \sqrt{\frac{1}{3 \sin \frac{\pi}{3} A}}}{\frac{L}{\sqrt{A}}}=2 \sqrt{\frac{1}{3 \sin \frac{\pi}{3}}} \approx 1.24
$$

$n_{0}<n_{1}$, which means the shape of the maximum number is rectangle

So far, we give our conclusion for this number model:
- the rectangle is the shape of potential maximum number

- From square to round, the number of regular polygon gradually decreases and approaches the number of round pans, which is the minimum.

\section{Result}

When heated under a certain temperature, the heat distribution over edges of different pans are studied. After numerical simulation, we found the points of maximum temperature in a rectangular pan are at the corners and the relative higher temperature always makes the food at corner overcooked. But as number of edges increases, heat distribution on a pan surface tends to be more uniform. Theoretically, marginal temperature distribution on a round pan reaches evenness. Therefore, use pans with more edges, there is a lower possibility for you to get overcooked food.

\section{References}

[1] Webb R L, Kim N H. Principle of enhanced heat transfer [M]. 2nd ed. Boca Raton: Taylor \& Francis, 2005:28.

[2] Cheng B, Tao W Q. Experimental study of R-152a film condensation on single horizontal smooth and enhanced tube $[\mathrm{J}]$. ASME J Heat transfer, 1994:116(1):266-270.

[3] Aziz A. The critical thickness of insulation [J]. Heat Transfer Engineering, 1997, 18(2):61-93. 